\title{
Columnar cell lesions and subsequent breast cancer risk: a nested case-control study
}

Sarah A Aroner ${ }^{1 *}$, Laura C Collins ${ }^{2}$, Stuart J Schnitt ${ }^{2}$, James L Connolly ${ }^{2}$, Graham A Colditz ${ }^{3}$, Rulla M Tamimi ${ }^{1,4}$

\begin{abstract}
Introduction: Histologic and genetic evidence suggests that at least some columnar cell lesions (CCL) of the breast represent precursor lesions in the low-grade breast neoplasia pathway. However, the risk of subsequent breast cancer associated with the presence of CCL in a benign breast biopsy is poorly understood.

Methods: The authors examined the association between the presence of $C C L$ and subsequent breast cancer risk in a nested case-control study of benign breast disease (BBD) and breast cancer within the Nurses' Health Studies (394 cases, 1,606 controls). Benign breast biopsy slides were reviewed by pathologists and CCL presence assessed. Logistic regression was used to compute odds ratios (ORs) and 95\% confidence intervals (Cls) for the association between $\mathrm{CCL}$ and breast cancer risk.

Results: Women with CCL (140 cases, 448 controls) had an increased risk of breast cancer compared with those without $\mathrm{CCL}(\mathrm{OR}=1.44,95 \% \mathrm{Cl}: 1.14$ to 1.83$)$, although this was attenuated and became non-significant after adjustment for the histologic category of $\mathrm{BBD}(\mathrm{OR}=1.20,95 \% \mathrm{Cl}: 0.94$ to 1.54$)$. $\mathrm{CCL}$ presence was associated with the greatest risk of breast cancer for those with nonproliferative $\mathrm{BBD}(\mathrm{OR}=1.36,95 \% \mathrm{Cl}: 0.79$ to 2.37$)$ and the lowest risk for those with atypical hyperplasia $(\mathrm{AH})(\mathrm{OR}=1.10,95 \% \mathrm{Cl}: 0.65$ to 1.87); however, this apparent heterogeneity in risk across BBD categories was not significant ( $P$ for interaction between CCL presence and BBD category $=0.77$ ).

Conclusions: These results provide evidence that $C C L$ may be an important marker of breast cancer risk in women with BBD but suggest that CCL do not increase breast cancer risk independently of concurrent proliferative changes in the breast.
\end{abstract}

\section{Introduction}

With the widespread adoption of screening mammography in the past several decades, columnar cell lesions (CCL) of the breast have become a frequent finding in breast biopsies. In fact, these lesions have been reported to be present in nearly half of biopsies performed for mammographic microcalcifications and have been identified as the source of three-quarters of biopsy-detected microcalcifications [1]. CCL is a broad designation that encompasses a wide range of histologic changes ranging from alterations of the epithelium with no architectural or cytologic atypia to changes resembling ductal carcinoma in situ (DCIS) [2]. Although these lesions have been recognized for some time, the diverse terminology

\footnotetext{
* Correspondence: sarah.aroner@channing.harvard.edu

'Department of Epidemiology, Harvard School of Public Health, 677

Huntington Avenue, Boston, MA 02115, USA Full list of author information is available at the end of the article
}

used to describe such lesions has hindered the understanding of their clinical significance. The myriad of names used to describe these lesions include "blunt duct adenosis" [3,4], "clinging carcinoma" [5,6], "columnar alteration with prominent apical snouts and secretions" [1], "atypical ductal cells with apocrine snouts" [7], "atypical cystic lobules" [8-10], and "well-differentiated DCIS with a clinging architecture" [11]. The increasing use of the term "columnar cell lesions" and the standardization of the classification scheme for these lesions [2] is facilitating the study of CCL as a potential breast cancer risk factor.

Some of the earliest evidence suggesting that CCL may be premalignant was their frequent detection near known precancerous and cancerous changes in the breast. Several studies have reported a high frequency of CCL and low-grade DCIS in the same breast $[1,8,12]$, with concurrent CCL and DCIS commonly occurring in
Ciomed Central 
the same or adjacent terminal duct lobular units [1]. Cooccurrence of CCL and low-grade invasive carcinomas, particularly tubular carcinoma, has also been observed [7,13-16]. The presence of CCL in proximity to invasive and non-invasive breast cancer suggests that CCL may have the potential to undergo malignant transformation, although CCL may simply be markers for other precancerous conditions in the breast.

Mounting histopathologic and molecular evidence indicates that CCL may indeed be early precursors to low-grade invasive breast carcinomas. CCL have been proposed as breast cancer precursors largely because of the cytologic and architectural similarities of more advanced CCL to atypical hyperplasia $(\mathrm{AH})$, a suspected precancerous abnormality, and DCIS, a well-established precursor to invasive carcinoma $[1,8,10]$. In many cases, the cellular features of advanced CCL so closely resemble those of low-grade DCIS that the distinction between the two lesions can be challenging $[1,8]$. Genetic alterations in CCL following a similar progression as the morphological changes have also been observed, and the mutations in the most advanced lesions have been found to be very similar to those in DCIS or invasive cancer [17-19]. Supporting these findings is evidence of an immunophenotypic link between CCL and breast carcinoma, with advanced CCL displaying an immunohistochemical profile similar to that of DCIS and low-grade invasive cancer [8,18,20-22].

Descriptive studies among patients with advanced $\mathrm{CCL}$, although unable to quantify the elevation in breast cancer risk associated with CCL, suggest that CCL are unlikely to be a strong risk factor for subsequent breast cancer. While Martel et al. did find some suggestion of an increased risk of breast cancer subsequent to CCL, as 9 out of 63 participants with advanced CCL (14.3\%) developed subsequent infiltrating carcinomas after eight years of follow-up [23], most other descriptive studies have found little evidence for an association between CCL and subsequent breast cancer. In fact, De Mascarel et al. found that none of 84 patients with advanced CCL alone developed subsequent breast cancer over a 10-year follow-up [24]. Similarly, no cases of invasive breast cancer were found among 59 patients with CCL followed for a median of 5.4 years in the European Organization for Research and Treatment of Cancer randomized clinical trial [11] or among 25 patients with CCL followed for 19.2 years in a retrospective analysis by Eusebi $e t$ al. [6]. Although these descriptive studies have provided insight into the natural history of BBD, most were limited by their small sample sizes and short follow-up, and their absence of a comparison group without CCL precludes assessment of the risk associated with CCL.

The very limited epidemiologic evidence on CCL suggests that CCL may be markers for concurrent atypical proliferative changes in the breast rather than breast cancer precursors [25-27]. Given the scarcity of epidemiologic data on the nature of these lesions, we undertook a study of CCL in relation to breast cancer risk using data from an established case-control study of benign breast disease (BBD) within the Nurses' Health Study (NHS) and the Nurses' Health Study II.

\section{Materials and methods \\ Study population}

The NHS is an ongoing prospective cohort study that began in 1976, when 121,700 female registered nurses between ages 30 and 55 years completed a mailed, selfadministered questionnaire regarding their health behaviors, lifestyle factors, and medical histories. Follow-up questionnaires have been sent to participants every two years to obtain updated information. The biennial questionnaires have assessed a variety of known and suspected risk factors for breast cancer, including history of BBD. On the 1976, 1978, and 1980 questionnaires, participants were asked if they had ever been diagnosed with fibrocystic or other BBD and whether this diagnosis had required hospitalization; from 1982 onward, the questionnaires have inquired specifically about BBD confirmed by biopsy. Deaths are reported by family members and the postal service, and regular searches of the computerized National Death Index are also conducted [28]. The NHS II is a separate cohort study consisting of 116,609 female registered nurses who were between the ages of 25 and 42 years when the study began in 1989. The follow-up methods used in this cohort are very similar to those for the original NHS. On each biennial questionnaire, participants have been asked if they have ever been diagnosed with BBD and, if yes, whether the diagnosis was confirmed by biopsy or aspiration. The follow-up rate for each two-year cycle has been greater than $90 \%$ of the original cohorts.

We conducted a nested case-control study to assess morphological changes in benign breast lesions and breast cancer risk among participants in the NHS and NHS II who had reported a previous diagnosis of BBD that was confirmed by biopsy. Within this subcohort, eligible cases were women who reported a first diagnosis of breast cancer between 1976 and the return of the 1996 questionnaire in the NHS or between 1989 and return of the 1995 questionnaire in the NHS II. Selfreported breast cancers were confirmed by review of medical records, and both invasive breast cancer and carcinoma in situ were included in the study. Eligible controls were women who completed the questionnaire for the same year that the breast cancer case was reported and had a previous diagnosis of biopsy-confirmed BBD, but were free from breast cancer; within each NHS cohort, they were matched to breast cancer 
cases on year of birth and year of diagnosis of BBD. We attempted to identify four matched controls for each case, but this was not always possible for logistical reasons. Informed consent was obtained from all participants. The study was approved by the Human Research Committee of Brigham and Women's Hospital, Boston, Massachusetts.

\section{Benign breast biopsy specimens}

Eligible cases and controls were contacted for permission to obtain their BBD pathology records and biopsy specimens, and specimens then were obtained from hospital pathology departments when possible. These methods have been described in detail elsewhere $[29,30]$. Briefly, $>70 \%$ of the 1,310 cases and 5,273 controls who originally were identified for the study confirmed the diagnosis and granted permission, and specimens subsequently were obtained for $52 \%$ of those who had granted their permission (465 cases and 1,939 controls). The primary reason given by hospital pathology departments for not sending specimens was that they had been destroyed or were no longer available (35\%) [29,30]. Biopsy slides were independently reviewed by one of two study pathologists (SJS, JLC) who were blinded to the participants' case or control status. The pathologists completed a detailed worksheet with information on the morphologic features of each specimen, and biopsies were then classified as nonproliferative, proliferative without atypia, or $\mathrm{AH}$, according to the criteria of Page et al. [31,32], which have been used in previous investigations in this cohort [29,33,34]. All biopsies, including bilateral biopsies, were classified according to the most severe changes present, and specimens with possible or definite AH were jointly reviewed by both pathologists. After excluding participants whose benign biopsy specimens were of poor quality or had no breast tissue, evidence of carcinoma in situ or invasive carcinoma, invalid dates of diagnosis, or insufficient information on laterality, there were a total of 395 cases and 1,610 controls $[29,30]$. Although we were successful in obtaining slides for only $30 \%$ of those originally identified, the success rate did not differ significantly between those with a subsequent breast cancer diagnosis and those without. In addition, the breast cancer risk factors for women from whom we were successful in obtaining pathology specimens were very similar to those for the women for whom we were unable to obtain specimens, suggesting that these women were missing at random.

During histologic review of benign breast biopsies for breast cancer cases reported in the 1976 through 1996 questionnaires in the NHS and in the 1991 through 1995 questionnaires in the NHS II and for matched controls, the presence of CCL was recorded on the pathology worksheets as "columnar alteration of lobules." At the time these benign breast biopsies were reviewed, CCL were not considered to be of particular interest and were usually not further stratified; thus, the categorization of these lesions as "columnar alteration of lobules" was in keeping with the standard of practice of the time. For the 42 cases and 116 controls from the NHS 1996 questionnaire cycle, presence of CCL subtypes (columnar cell change, columnar hyperplasia, and flat epithelial atypia) was assessed rather than the overall presence of CCL. These women with only subtype information were re-categorized by presence or absence of CCL to allow their inclusion in analyses. After exclusion of five subjects having lesions without adjacent tissue for evaluation, the final sample for this analysis included 394 cases and 1,606 controls. Given the small number of cases and controls in which CCL were further classified with regard to the presence or absence of atypia, CCL were considered as a single group for these analyses.

\section{Statistical analysis}

The distributions of breast cancer risk factors were examined according to CCL presence among the controls, adjusting for age at benign biopsy when appropriate. Unconditional logistic regression was used to estimate odds ratios (ORs) and 95\% confidence intervals (95\% CIs) for breast cancer risk according to CCL presence. Unconditional rather than conditional logistic regression was used because this analytic approach allowed us to use all cases and controls for whom we had histologic information. We first adjusted only for the matching factors, which were age at breast cancer diagnosis or index date ( $<45$ years, 45 to 49 years, 50 to 54 years, 55 to 59 years, $\geq 60$ years), year of benign breast biopsy (before 1970, 1970 to 1979,1980 to 1989 , 1990 and later), and time since benign biopsy (years from BBD diagnosis to the breast cancer diagnosis or index date, as a continuous variable). In another set of models, additional adjustment was made for histologic category of BBD (nonproliferative, proliferative without atypia, or $\mathrm{AH}$ ), which is a strong predictor of breast cancer risk [31]. We also jointly classified women according to histologic category and CCL presence to examine whether the association of CCL with breast cancer risk differed by category of BBD; a likelihood ratio test was used to test for interaction.

The following covariates were considered as potential confounding factors: first-degree family history of breast cancer (yes, no), age at menarche ( $<12$ years, 12 years, 13 years, $\geq 14$ years), menopausal status (premenopausal, postmenopausal, dubious/unsure), jointly classified parity and age at first birth (nulliparous, 1 to 2 children and $<25$ years, 1 to 2 children and 25 to 29 years, 1 to 2 children and $\geq 30$ years, $\geq 3$ children and $<25$ years, $\geq 3$ children and 
$\geq 25$ years), BMI at age $18(<21,21$ to $22.9,23$ to 24.9 , $25+\mathrm{kg} / \mathrm{m}^{2}$ ), postmenopausal hormone use (premenopausal, postmenopausal never, ever), and oral contraceptive use (never, ever). Because BMI at age 18 appeared to be more strongly related to breast cancer risk than current BMI, we chose to consider adjustment for BMI at age 18 and change in weight since age 18 rather than current BMI. Individual adjustment for these covariates did not change the estimated OR substantially, nor did simultaneous adjustment for the four factors having the strongest influence on the OR (BMI at age 18, family history of breast cancer, postmenopausal hormone use, and jointly classified parity/age at first birth). We examined relationships between CCL and estrogen receptor (ER) status, grade, and ipsilaterality in additional unconditional logistic regression models. All statistical analyses were performed using the SAS software package (version 9.1; SAS Institute, Cary, NC, USA). All tests were two-sided, with $P<0.05$ indicating statistical significance.

\section{Results}

The mean age at benign breast biopsy among all participants was 43.7 years ( $S D=10.0$ years), and the mean time since biopsy was 10.0 years (SD $=7.2$ years). Compared with women with no CCL, women with CCL were older at the time of their biopsy and had less time since their biopsies (Table 1). Women with and without CCL were generally similar with regard to their distribution of breast cancer risk factors, although women with CCL were slightly leaner than those without CCL and were less likely to have used postmenopausal hormones. Histologic subtype of BBD was strongly associated with presence of CCL, with women having CCL much more likely to have proliferative disease and $\mathrm{AH}$ compared with women without CCL $(P<0.0001)$. We also assessed breast cancer risk factors by case-control status and found that established breast cancer risk factors were associated with breast cancer in our study population, although most of these associations were non-significant. Compared with controls, cases had a higher prevalence of family history of breast cancer, lower mean parity and older mean age at first birth among parous women, higher mean alcohol consumption, and a higher prevalence of postmenopausal hormone use (see Supplementary table S1 in Additional file 1).

In the multivariate logistic regression models controlling for matching factors only (Table 2), women with CCL had a significantly higher risk of breast cancer compared with those without CCL $(\mathrm{OR}=1.44$; 95\% CI, 1.14 to 1.83). However, after additional adjustment for histologic category of benign breast disease, this association became non-significant $(\mathrm{OR}=1.20 ; 95 \% \mathrm{CI}, 0.94$ to 1.54). These associations were very similar when only invasive breast cancer cases were included $(\mathrm{OR}=1.44$;
Table 1 Characteristics by CCL status among controls with BBD*

\begin{tabular}{|c|c|c|}
\hline & $\mathrm{CCL}$ & No CCL \\
\hline No. of controls (\%) & $\begin{array}{l}448 \\
(27.9)\end{array}$ & $\begin{array}{l}1,158 \\
(72.1)\end{array}$ \\
\hline \multicolumn{3}{|l|}{ Means } \\
\hline Age at BBD biopsy, years & 45.1 & 43.3 \\
\hline Year of BBD biopsy (median) & 1979 & 1979 \\
\hline Time since BBD biopsy, years & 9.3 & 10.1 \\
\hline No. of BBD biopsy slidest & 5.7 & 5.5 \\
\hline Body mass index, $\mathrm{kg} / \mathrm{m}^{2}+$ & 24.2 & 25.0 \\
\hline Body mass index at age $18, \mathrm{~kg} / \mathrm{m}^{2}+$ & 20.5 & 21.1 \\
\hline Weight gain after age $18, \mathrm{~kg} \dagger$ & 10.0 & 10.7 \\
\hline Age at menarche, yearst & 12.7 & 12.6 \\
\hline Age at first birth, years (parous only) + & 24.9 & 24.9 \\
\hline Alcohol intake, g/day $\dagger$ & 4.2 & 4.0 \\
\hline Parity (parous only)† & 3.0 & 3.0 \\
\hline \multicolumn{3}{|l|}{ Percentages } \\
\hline Premenopausalt & 36.0 & 35.5 \\
\hline Paroust & 91.9 & 92.4 \\
\hline $\begin{array}{l}\text { First degree family history of breast } \\
\text { cancert }\end{array}$ & 19.0 & 17.6 \\
\hline Ever postmenopausal hormone uset & 32.0 & 37.3 \\
\hline Ever oral contraceptive uset & 45.1 & 49.6 \\
\hline \multicolumn{3}{|l|}{ Histologic category of $\mathrm{BBD}+$} \\
\hline Nonproliferative & 21.5 & 44.4 \\
\hline Proliferative without atypia & 61.8 & 48.4 \\
\hline Atypical hyperplasia & 16.8 & 7.2 \\
\hline
\end{tabular}

$\mathrm{CCL}$ indicates columnar cell lesions; $\mathrm{BBD}$, benign breast disease.

*Unless otherwise specified, all variables correspond to the time period immediately prior to the index date.

†Means and percentages were standardized to the age distribution of the controls at the time of benign biopsy, in five-year categories.

95\% CI, 1.11 to 1.86 with adjustment for matching factors only and OR $=1.18 ; 95 \% \mathrm{CI}, 0.90$ to 1.55 with additional adjustment for histologic category of BBD).

When women were jointly classified according to histologic category and CCL presence, all groups had an elevated breast cancer risk compared with the reference category, nonproliferative BBD and no CCL. Women with both $\mathrm{AH}$ and $\mathrm{CCL}$ were at highest risk $(\mathrm{OR}=4.37$; 95\% CI, 2.81 to 6.81 ), but this risk was not significantly different from that among those with AH without CCL. Within all BBD histologic categories, breast cancer risk was elevated for women with CCL, with the risk higher for those with less severe BBD (nonproliferative BBD: $\mathrm{OR}=1.36$, 95\% CI: 0.79 to 2.37 ; proliferative BBD without atypia: $\mathrm{OR}=1.21,95 \% \mathrm{CI}: 0.87$ to 1.68 ; and $\mathrm{AH}$ : $\mathrm{OR}=1.10,95 \% \mathrm{CI}: 0.65$ to 1.87 ; adjustment for matching factors only). However, there was no evidence of an interaction between CCL and BBD histologic category when either three histologic categories were used $(P=0.77)$ or when proliferative disease without atypia and $\mathrm{AH}$ were collapsed into a single category $(P=0.93)$. 
Table 2 ORs and 95\% Cls for CCL and breast cancer risk among participants with BBD

\begin{tabular}{lllll}
\hline & Cases & Controls & OR $(\mathbf{9 5 \%} \mathbf{C l})^{*}$ & OR $(\mathbf{9 5} \% \mathbf{C l})^{* *}$ \\
\hline $\begin{array}{l}\text { CCL status } \\
\quad \text { No CCL }\end{array}$ & 254 & 1,158 & 1.0 (reference) & $1.0($ reference) \\
$\quad$ CCL & 140 & 448 & $1.44(1.14,1.83)$ & $1.37(1.06,1.76)$ \\
CCL status, adjusted for BBD histologic category & & & \\
$\quad$ No CCL & 254 & 1,158 & 1.0 (reference) & $1.0($ reference) \\
$\quad$ CCL & 140 & 448 & $1.20(0.94,1.54)$ & $1.16(0.89,1.50)$ \\
Jointly classified BBD/CCL histologic category & & & \\
$\quad$ Nonproliferative, no CCL & 80 & 518 & $1.0($ reference) & $1.0($ reference) \\
$\quad$ Nonproliferative, CCL & 19 & 93 & $1.33(0.77,2.29)$ & $1.24(0.69,2.22)$ \\
$\quad$ Proliferative without atypia, no CCL & 125 & 558 & $1.46(1.08,1.99)$ & $1.38(1.00,1.91)$ \\
$\quad$ Proliferative without atypia, CCL & 74 & 277 & $1.81(1.27,2.58)$ & $1.63(1.12,2.38)$ \\
$\quad$ Atypical hyperplasia, no CCL & 49 & 82 & $4.26(2.75,6.59)$ & $4.04(2.57,6.36)$ \\
$\quad$ Atypical hyperplasia, CCL & 47 & 78 & $4.37(2.81,6.81)$ & $4.20(2.63,6.70)$ \\
\hline
\end{tabular}

OR indicates odds ratio; $95 \% \mathrm{Cl}, 95 \%$ confidence interval.

*Adjusted for age, year of BBD diagnosis, and time since BBD biopsy.

**Adjusted for the above-mentioned factors and body mass index at age 18, weight change between age 18 and diagnosis/index date, family history of breast cancer, postmenopausal hormone use, and jointly classified parity/age at first birth.

There was some suggestion that women with CCL had a higher risk of ER positive and ipsilateral breast cancer compared with women without CCL, but these associations were not significant after adjustment for BBD histologic category. Breast cancer grade appeared to be unrelated to CCL status.

\section{Discussion}

Our results in this established nested-case control study of BBD point to a modestly increased risk of breast cancer among women with CCL. However, it appeared that most of this elevation in risk was due to the close association between CCL and proliferative disease, which itself is an established risk factor for subsequent breast cancer [31]. Despite the apparent lack of an independent association between CCL and breast cancer, there was an indication that CCL may influence breast cancer risk among women with nonproliferative disease.

Few previous epidemiologic analyses have evaluated the risk of subsequent breast cancer following detection of CCL, but the magnitude of risk associated with the presence of CCL found in our study is consistent with that reported by Boulos et al. in a large retrospective cohort study among women in the Nashville Breast Cohort. Similar to our study, the Nashville study found a nearly $50 \%$ elevation in breast cancer risk associated with CCL ( $R R=1.47 ; 95 \% \mathrm{CI}: 1.0$ to 2.2 ), although this analysis excluded those with $\mathrm{AH}$, who have been shown to be at highest risk of subsequent breast cancer. Given the strong correlation between $\mathrm{CCL}$ and concurrent $\mathrm{AH}$ found by Boulos et al. $(P<0.0005)$, it is possible that the relative risk for presence of CCL would have been somewhat higher had women with $\mathrm{AH}$ been included in the main analysis. As in our study, Boulos et al. found that the elevated risk of breast cancer among those with $\mathrm{AH}$ was not further increased among those who additionally had CCL, suggesting that CCL are not an independent breast cancer risk factor among women with $\mathrm{AH}$ [25]. Another recent analysis by Kabat et al. that assessed subsequent breast cancer risk from a broad range of benign breast lesions found a non-significantly elevated risk of breast cancer among a small number of women with multiple CCL compared with those with non-proliferative disease and no breast pathology ( $R R=$ 1.72, 95\% CI: 0.87 to 3.41 ), although no increased risk was found for focal CCL (RR $=0.81,95 \%$ CI: 0.47 to 1.39) [27]. However, it is difficult to compare our results with those from the study by Kabat et al. because this study used a different pathologic classification scheme in which CCL were considered a proliferative disease rather than a separate entity that could exist in conjunction with either nonproliferative or proliferative BBD.

Our study is the largest nested case-control study of $\mathrm{CCL}$ and breast cancer to date and therefore yields important clues into the relationship between presence of CCL and breast cancer risk. However, given the heterogeneity of CCL, it is possible that assessing risk of breast cancer for all CCL subtypes combined masks an effect of advanced CCL subtypes, which are less common. Histopathologic and molecular evidence suggests that CCL displaying low-grade cytologic atypia, recently termed "flat epithelial atypia" (FEA) by the World Health Organization Working Group on the Pathology and Genetics of Tumors of the Breast [35], may be a direct precursor to low-grade DCIS, while the more common non-atypical CCL are not thought to be precancerous [36].

However, the malignant potential of FEA remains equivocal, as descriptive and epidemiologic studies fail 
to support molecular findings, just as they do for overall CCL risk. Most descriptive follow-up studies among women with what would now be classified as FEA, including one with an average follow-up of nearly 20 years, have found progression to invasive carcinoma to be uncommon $[6,11,23,24]$. Three previous observational studies have assessed CCL subtype-specific risks, but all had limited power to detect associations for FEA due to small numbers of women with this subtype. Two of these studies did find an increased risk among women with atypical CCL compared with those having nonproliferative disease [27] or with all women without atypical CCL [26], but results were non-significant and confidence intervals were very wide, as one study had 19 women with atypical CCL [26] and the other had only 6 [27]. The largest of the three studies found no evidence of an increased breast cancer risk associated with "columnar cell lesions with atypia" among women without $\mathrm{AH}$. This study did indicate that risk may be increased for those having CCL with hyperplasia, although this could have been a chance finding due to the small number of women with each CCL subtype even in this larger analysis [25].

Although we considered performing a CCL subtypespecific analysis, information on subtype (columnar cell change, columnar hyperplasia, and FEA) was collected for only 157 women whose breast cancer or index date was reported on the 1996 NHS questionnaire. We chose not to re-review the biopsy slides for the presence of FEA, as prevalence of FEA appeared very low from the 1996 questionnaire cycle, with only 4 of the women out of 53 with CCL from this cycle having FEA. Because FEA is a rare occurrence, many thousands of breast biopsies would be needed for adequate power to study its relationship with subsequent breast cancer. Moreover, to study the association between FEA and breast cancer independent of $\mathrm{AH}$, an adequate number of women with FEA but not AH would be required. This would only be possible in an extremely large study, as FEA commonly occurs in conjunction with $\mathrm{AH}$. Even in the largest previous epidemiologic study of CCL by Boulos et al., only 52 biopsies contained FEA without AH [25].

The strengths of the study include its prospective design, the confirmation of breast cancer cases through review of medical records, the centralized pathology review of specimens from benign biopsies before the development of breast cancer, and the detailed information on other breast cancer risk factors. Furthermore, ours is the first observational study to compare the risk of subsequent breast cancer by BBD histologic category. Although power was limited to test for heterogeneity by histologic category, our findings suggest that CCL may be a marker of increased risk among those with nonproliferative disease. Such a differential effect of CCL on breast cancer risk across BBD categories could have important implications for clinical management of patients with CCL, especially given that women with non-proliferative disease are typically considered to be at no elevated risk for breast cancer compared with women from the general population.

\section{Conclusions}

In light of these findings, large epidemiologic studies assessing CCL subtype-specific risks are needed to inform clinical guidelines for the management of CCL. In particular, better understanding is needed as to whether CCL with atypia in the absence of more advanced lesions require treatment similar to that for DCIS. Additionally, adequately powered epidemiologic studies might help elucidate whether CCL are of concern only among women without proliferative disease. Regardless of whether particular CCL subtypes are simply markers for premalignant changes in the breast or precancerous histologic changes themselves, recognition of these lesions is nevertheless important, as detection of CCL via biopsy should prompt additional scrutiny for other pathologic changes in breast tissue that may signal the early development of DCIS or invasive carcinoma.

\section{Additional material}

Additional file 1: Supplementary table S1. A Word document containing a table that lists the means and percentages of characteristics by case-control status among participants with benign breast disease (BBD).

\section{Abbreviations}

$\mathrm{AH}$ : atypical hyperplasia; BBD: benign breast disease; BMl: body mass index; CCL: columnar cell lesions; Cl: confidence interval; DCIS: ductal carcinoma in situ; ER: estrogen receptor; FEA: flat epithelial atypia; NHS: Nurses' Health Study; OR: odds ratio.

\section{Acknowledgements}

Funding and/or support came from Public Health Service Grants CA046475, CA087969, CA050385, and SPORE in Breast Cancer CA089393 from the National Cancer Institute, National Institutes of Health, Department of Health and Human Services and the Breast Cancer Research Foundation, and the American Cancer Society (to G. A. Colditz).

\section{Author details}

${ }^{1}$ Department of Epidemiology, Harvard School of Public Health, 677 Huntington Avenue, Boston, MA 02115, USA. ²Department of Pathology, Beth Israel Deaconess Medical Center and Harvard Medical School, 330 Brookline Avenue, Boston, MA 02115, USA. ${ }^{3}$ Department of Surgery, Washington University School of Medicine, 660 S. Euclid Avenue, St. Louis, MO 63110, USA. ${ }^{4}$ Channing Laboratory, Department of Medicine, Brigham and Women's Hospital and Harvard Medical School, 181 Longwood Avenue, Boston, MA 02115, USA.

\section{Authors' contributions}

SA participated in the study design, data analysis and interpretation, and manuscript drafting. LC participated in the study design, data collection, and manuscript revision. SS and JC reviewed the BBD biopsy slides and participated in the study design and manuscript revision. GC participated in 
the study design, data collection, and manuscript revision. RT participated in study design, data collection, manuscript revision, and study supervision. All authors read and approved the final manuscript.

\section{Competing interests}

The authors declare that they have no competing interests.

Received: 12 May 2010 Revised: 21 July 2010 Accepted: 6 August 2010 Published: 6 August 2010

\section{References}

1. Fraser UL, Raza S, Chorny K, Connolly JL, Schnitt SJ: Columnar alteration with prominent apical snouts and secretions: a spectrum of changes frequently present in breast biopsies performed for microcalcifications. Am J Surg Pathol 1998, 22:1521-1527.

2. Schnitt SJ, Vincent-Salomon A: Columnar cell lesions of the breast. Adv Anat Pathol 2003, 10:113-124.

3. Foote FW, Stewart FW: Comparative Studies of Cancerous Versus Noncancerous Breasts. Ann Surg 1945, 121:197-222.

4. Lanyi M, Citoler P: The differential diagnosis of microcalcification. Microcyst (blunt duct) adenosis (author's transl). Rofo 1981, 134:225-231.

5. Azzopardi J: Problems in Breast Pathology Philadelphia: WB Saunders 1979.

6. Eusebi V, Feudale E, Foschini MP, Micheli A, Conti A, Riva C, Di Palma S, Rilke F: Long-term follow-up of in situ carcinoma of the breast. Semin Diagn Pathol 1994, 11:223-235.

7. Goldstein NS, O'Malley BA: Cancerization of small ectatic ducts of the breast by ductal carcinoma in situ cells with apocrine snouts: a lesion associated with tubular carcinoma. Am J Clin Pathol 1997, 107:561-566.

8. Oyama T, lijima K, Takei H, Horiguchi J, lino Y, Nakajima T, Koerner F: Atypical cystic lobule of the breast: an early stage of low-grade ductal carcinoma in-situ. Breast Cancer 2000, 7:326-331.

9. Koerner FC, Oyama T, Maluf H: Morphological observations regarding the origins of atypical cystic lobules (low-grade clinging carcinoma of flat type). Virchows Arch 2001, 439:523-530.

10. Oyama T, Maluf H, Koerner F: Atypical cystic lobules: an early stage in the formation of low-grade ductal carcinoma in situ. Virchows Arch 1999, 435:413-421.

11. Bijker N, Peterse JL, Duchateau L, Julien JP, Fentiman IS, Duval C, Di Palma S, Simony-Lafontaine J, de Mascarel I, van de Vijver MJ: Risk factors for recurrence and metastasis after breast-conserving therapy for ductal carcinoma-in-situ: analysis of European Organization for Research and Treatment of Cancer Trial 10853. J Clin Oncol 2001, 19:2263-2271.

12. Page DL, Kasami M, Jensen RA: Hypersecretory hyperplasia with atypia in breast biopsies. What is the proper level of clinical concern? Pathol Case Reviews 1996, 1:36-40.

13. Abdel-Fatah TM, Powe DG, Hodi Z, Lee AH, Reis-Filho JS, Ellis IO: High frequency of coexistence of columnar cell lesions, lobular neoplasia, and low grade ductal carcinoma in situ with invasive tubular carcinoma and invasive lobular carcinoma. Am J Surg Pathol 2007, 31:417-426.

14. Brandt SM, Young GQ, Hoda SA: The "Rosen Triad": tubular carcinoma, lobular carcinoma in situ, and columnar cell lesions. Adv Anat Pathol 2008, 15:140-146.

15. Fernandez-Aguilar S, Simon P, Buxant F, Simonart T, Noel JC: Tubular carcinoma of the breast and associated intra-epithelial lesions: a comparative study with invasive low-grade ductal carcinomas. Virchows Arch 2005, 447:683-687.

16. Rosen PP: Columnar cell hyperplasia is associated with lobular carcinoma in situ and tubular carcinoma. Am J Surg Pathol 1999, 23:1561.

17. Moinfar F, Man YG, Bratthauer GL, Ratschek M, Tavassoli FA: Genetic abnormalities in mammary ductal intraepithelial neoplasia-flat type ("clinging ductal carcinoma in situ"): a simulator of normal mammary epithelium. Cancer 2000, 88:2072-2081.

18. Simpson PT, Gale T, Reis-Filho JS, Jones C, Parry S, Sloane JP, Hanby A, Pinder SE, Lee AH, Humphreys S, Ellis IO, Lakhani SR: Columnar cell lesions of the breast: the missing link in breast cancer progression? A morphological and molecular analysis. Am J Surg Pathol 2005, 29:734-746.

19. Dabbs DJ, Carter G, Fudge M, Peng Y, Swalsky P, Finkelstein S: Molecular alterations in columnar cell lesions of the breast. Mod Pathol 2006, 19:344-349.

20. Dessauvagie BF, Zhao W, Heel-Miller KA, Harvey J, Bentel JM: Characterization of columnar cell lesions of the breast: immunophenotypic analysis of columnar alteration of lobules with prominent apical snouts and secretions. Hum Pathol 2007, 38:284-292.

21. Tremblay G, Deschenes J, Alpert L, Quenneville LA: Overexpression of estrogen receptors in columnar cell change and in unfolding breast lobules. Breast J 2005, 11:326-332.

22. Lee S, Mohsin SK, Mao S, Hilsenbeck SG, Medina D, Allred DC: Hormones, receptors, and growth in hyperplastic enlarged lobular units: early potential precursors of breast cancer. Breast Cancer Res 2006, 8:R6.

23. Martel M, Barron-Rodriguez P, Tolgay Ocal I, Dotto J, Tavassoli FA: Flat DIN 1 (flat epithelial atypia) on core needle biopsy: 63 cases identified retrospectively among 1,751 core biopsies performed over an 8-year period (1992-1999). Virchows Arch 2007, 451:883-891.

24. de Mascarel I, MacGrogan G, Mathoulin-Pelissier S, Vincent-Salomon A, Soubeyran I, Picot V, Coindre JM, Mauriac L: Epithelial atypia in biopsies performed for microcalcifications. practical considerations about 2,833 serially sectioned surgical biopsies with a long follow-up. Virchows Arch 2007, 451:1-10.

25. Boulos FI, Dupont WD, Simpson JF, Schuyler PA, Sanders ME, Freudenthal ME, Page DL: Histologic associations and long-term cancer risk in columnar cell lesions of the breast: a retrospective cohort and a nested case-control study. Cancer 2008, 113:2415-2421.

26. Shaaban AM, Sloane JP, West CR, Moore FR, Jarvis C, Williams EM, Foster CS: Histopathologic types of benign breast lesions and the risk of breast cancer: case-control study. Am J Surg Pathol 2002, 26:421-430.

27. Kabat GC, Jones JG, Olson N, Negassa A, Duggan C, Ginsberg M, Kandel RA, Glass AG, Rohan TE: A multi-center prospective cohort study of benign breast disease and risk of subsequent breast cancer. Cancer Causes Control 2010, 21:821-828.

28. Stampfer MJ, Willett WC, Speizer FE, Dysert DC, Lipnick R, Rosner B, Hennekens CH: Test of the National Death Index. Am J Epidemiol 1984 119:837-839.

29. Collins LC, Baer HJ, Tamimi RM, Connolly JL, Colditz GA, Schnitt SJ: Magnitude and laterality of breast cancer risk according to histologic type of atypical hyperplasia: results from the Nurses' Health Study. Cancer 2007, 109:180-187.

30. Collins LC, Baer HJ, Tamimi RM, Connolly JL, Colditz GA, Schnitt SJ: The influence of family history on breast cancer risk in women with biopsyconfirmed benign breast disease: results from the Nurses' Health Study. Cancer 2006, 107:1240-1247.

31. Schnitt SJ, Connolly JL: Pathology of benign breast disorders. Diseases of the Breast Philadelphia: Lippincott Williams \& WilkinsHarris JR, Lippman ME, Morrow M, Osborne CK, 3 2004, 69-86.

32. Page DL, Dupont WD, Rogers LW, Rados MS: Atypical hyperplastic lesions of the female breast. A long-term follow-up study. Cancer 1985 55:2698-2708.

33. Marshall LM, Hunter DJ, Connolly JL, Schnitt SJ, Byrne C, London SJ, Colditz GA: Risk of breast cancer associated with atypical hyperplasia of lobular and ductal types. Cancer Epidemiol Biomarkers Prev 1997, 6:297-301.

34. London SJ, Connolly JL, Schnitt SJ, Colditz GA: A prospective study of benign breast disease and the risk of breast cancer. JAMA 1992 267:941-944.

35. Tavassoli FA, Hoefler $\mathrm{H}$, Rosai J: Intraductal proliferative lesions. World Health Organization Classification of Tumours Pathology and Genetics of Tumours of the Breast and Female Genital Organs Lyon, France: IARC PressTavassoli FA, Devilee P 2003, 63-73.

36. Jara-Lazaro AR, Tse GM, Tan PH: Columnar cell lesions of the breast: an update and significance on core biopsy. Pathology 2009, 41:18-27.

\section{doi:10.1186/bcr2624}

Cite this article as: Aroner et al:: Columnar cell lesions and subsequent breast cancer risk: a nested case-control study. Breast Cancer Research 2010 12:R61. 